\title{
Effects of Training Parameters on Neural Network Parallel Interference Canceller for CDMA
}

\author{
Jahan Rosemary Joseph and Anish Francis
}

\begin{abstract}
Reduction of multiple access interference is occupying great importance in wireless communication using code division multiple access (CDMA). Different interference cancellation schemes were developed and neural network based canceller is one of them. This paper introduces the effect of training parameters on achieving optimum performance on a multi stage parallel interference canceller (PIC) based on neural network.
\end{abstract}

Index Terms-BER, CDMA, LM algorithm, neural network.

\section{INTRODUCTION}

Mobile cellular communication is at its high reach these days. Direct Sequence Code Division Multiple Access (DS-CDMA) based wireless communication, undoubtedly one of the best among the different wireless technologies. The attractions of CDMA are because of its antimultipath capabilities, high security etc compared to other existing multiple access technologies.

But the major problem with the code division multiple access(CDMA) technologies is the multiple access interference (MAI) due to the presence of signals occupying the channel at all frequencies and time[1]. MAI reduces the channel capacity and affects the detection performance. This problem is solved to a great extent by using multi user detection(MUD) techniques[2]. But the computational complexity of the normal MUD technique affects performance for realistic number of users. The suboptimal detectors solve the problem to a great extent[3]. Neural network based parallel interference cancellers (PIC) are members of this class of suboptimal detectors. This paper investigates the effect of training parameters, on detector performance. A multitude of study was conducted on this area based by changing various parameters and observations were found regarding better performance of the detector by the changes.

The first neural network work based suboptimal detectors were first proposed by Azhang[4]. This classical paper ignited the research on neural network receivers and was followed by several other works [5]. Successive interference cancellers [6] and Parallel interference cancellers were proposed based on the neural network. The software programs were relied for simulating the multiuser

Manuscript received December 29, 2012; revised February 23, 2013.

Jahan Rosemary Joseph is with the Baselios Thomas I Catholicose College of Engineering and Technology, Kerala, India (e-mail: jahanrosemary@gmail.com).

Anish Francis is with the Kerala State Electricity Board(KSEB), Kerala, India. (e-mail: anishfran@gmail.com). environment required for CDMA system. This is emphasized in the classic paper of Darius Divsalar on improved parallel interference cancellation for CDMA [7]. This paper closely follows the above paper and [8]. The authors of [8], employed neural network between the matched filter stage and PIC for obtaining better bit error rate (BER) performance. In this paper, we attempts to change the training parameters of the inserted neural network stage and obtain high performance without changing the number of stages of PIC. A multitude of simulations were conducted for different parameters like signal to noise ratio (SNR), number of neurons and training ratios.

The paper is organized as follows. In the section II we explain the CDMA model briefly which is followed by a short introduction to the neural networks (section III) which is employed in our study. Finally, a greater part of the paper is dedicated to the explanation of simulation (section IV).

\section{CDMA MODEL}

The model of the synchronous system is shown in the Fig. 1. Each user's data are spread with gold code sequence for better correlation performance. The CDMA transmitter output is represented as

$$
y(t)=\sum_{k}^{1} a_{k} b_{k} s_{k}(t)+n(t)
$$

Here $a$ represents the received bit, $b$ represents the antipodal input bits, and $S$ represents the signature sequence $n$ represents the additive white Gaussian noise. Gold code sequence is used for spreading the user data [9].

$$
S_{k}(t)=\sum_{k=0}^{N-1} a_{k} p_{T}\left(t-k T_{c}\right)
$$

At the other end the signal is received by a matched filter bank. This output is specified by the equation

$$
y_{k}=A_{k} b_{k}+\sum_{j=1, \neq k}^{k} A_{k} b_{k} \rho_{k}+n_{k}
$$

The first part of the above equation represents, desired output, others are MAI and noise [10]. The purpose of the interference canceller is to detect this MAI and nullify it. In the Serial Interference Canceller (SIC), most powerful user's bit is assumed as correct and an order of power levels is set as criteria for correction. But SIC has large time delay. 


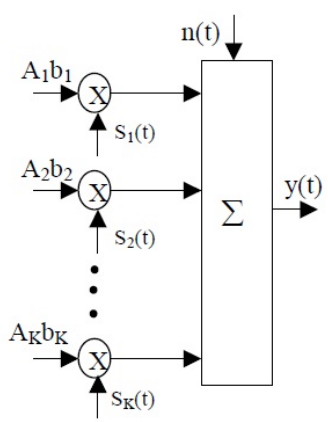

Fig. 1. The transmitter.

In the PIC correction process is done simultaneously for all users uniformly in one bit period. For better performance multi stage PICs are considered [11].

In this work we inserted the neural network block between the matched filter block and the PIC stages. The modified receiver side is shown in Fig. 2

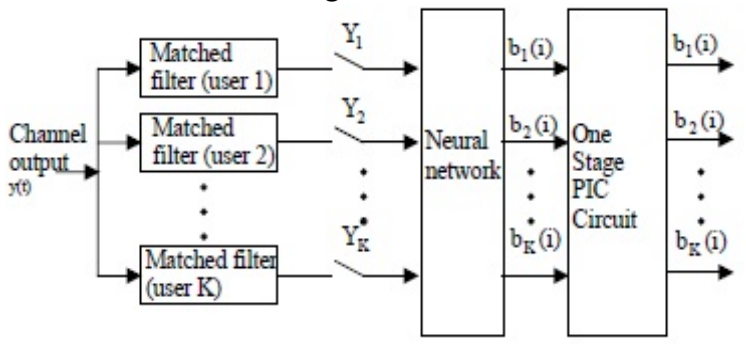

Fig. 2. Neural network receiver

\section{NEURAL NETWORKS}

Neural networks imitate nervous systems found in biological organisms. It is built of data processing units (neurons) connected via adjustable connection weights. Neurons are arranged in layers, an input layer, hidden layer(s), and an output layer. There is no specific rule that dictates the number of hidden layers. The function is mainly based on the connections between different neurons. In the input layer, each neuron is designated to one of the input parameters. The network can work as pattern detector, function approximator and so on. The network learns to adjust its weight adaptively by certain algorithm. This is done in 3 stages, training, validation and testing [12]-[13]. A neural network detection network for 2 users is shown in Fig. 3.

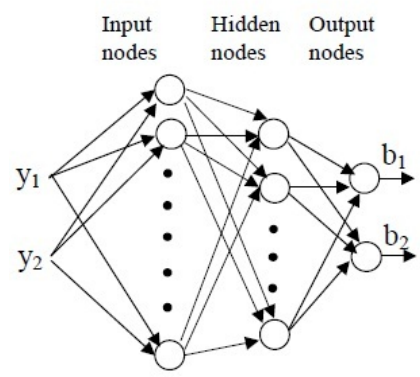

Fig. 3. Neural network

For working as a receiver the number of nodes is set as number of users. The matched filter output is set as the input to the neural network. The performance of the neural network as a detector for multiple access technique and the abilities of the simulating environment to mimic the realistic communication is given in [2]. The network is trained with Levenberg-Marquardt algorithm [14]-[15] for back propagation in the present work.

\section{SimULATION RESUlTS}

In the present work MATLAB neural network tool box [16] was used to simulate the CDMA system. We used a volume of 500 bits for running the simulations.

The training parameters we changed were number of hidden neurons and the training ratios. The ratio is the size of data used for training, testing and validation of the neural network. We used three ratios, 60:30:10, 80:20:10 and 90:5:5.The ratios are denoted A, B and C. we also tested the number of neurons up to 30 in various results. The results were compared with normal PIC receiver performance for various numbers of stages.

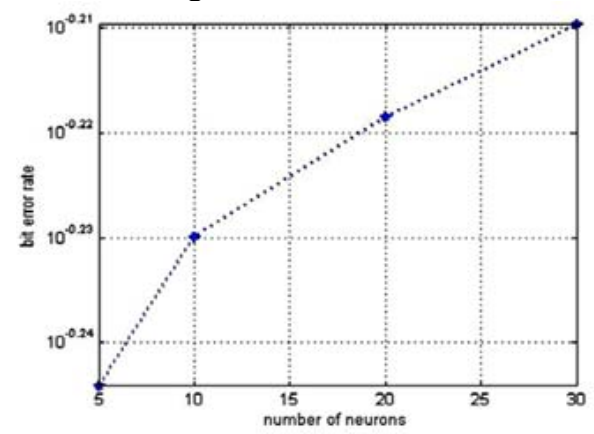

Fig. 4. BER versus number of hidden neurons

In the simulations, the first step was to find the optimum number of neutrons for good BER. We adopted a trial and error approach, for a 60:30:10 ratio (ratio A) with constant SNR of $3 \mathrm{~dB}$. We found that BER increases and then decreases but the optimum is around in 20. Hence for the succeeding tests we fixed the number of hidden neurons as 20.The plot for above test is shown in Fig. 4.

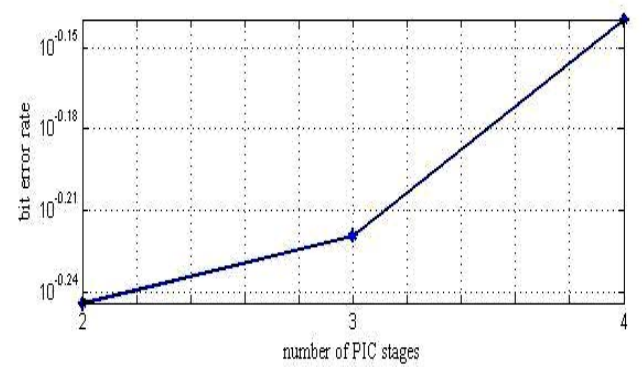

Fig. 5. BER versus number of stages, for ratio B

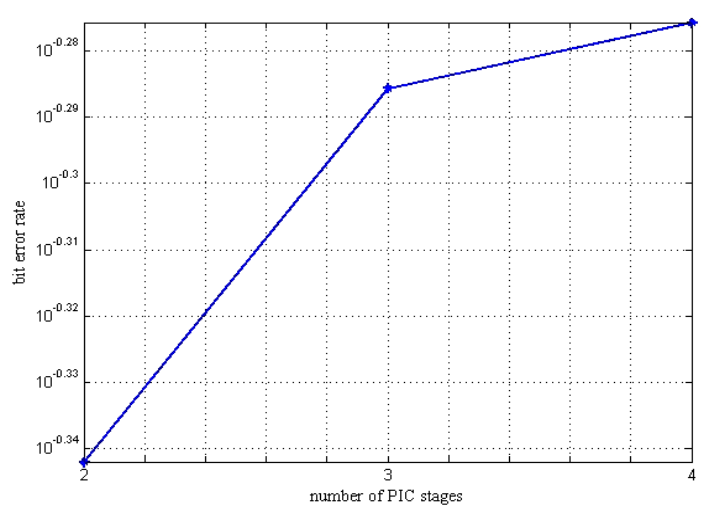

Fig. 6. BER versus number of stages, for ratio $\mathrm{C}$

Then simulations were conducted for other ratios, B and C, keeping this number of neurons constant. The results are 
shown in Fig. 5 and Fig. 6.

The above results show that better BER performance for ratio $\mathrm{C}$ over other ratios for same number of stages. This is more correct when the number of stages is lower. Example is Stage 2.

We also studied the variations of MSE and correlation coefficient in several trials. The results for ratio $\mathrm{A}$ is shown in Table I. A lower MSE value is desirable and for $\mathrm{R}$, a value closer to one is required for better performance.

TABLE I: MSE AND R VALUES FOR RATIO A

\begin{tabular}{llll} 
Network building stage & Best MSE & Worst MSE & Best R \\
\hline Training & 1.402 & 2.10 & $2.36 \mathrm{e}^{-1}$ \\
Testing & 1.693 & 3.064 & $3.46 \mathrm{e}^{-1}$ \\
Validation & 0.84 & 2.27 & $4.078 \mathrm{e}^{-1}$ \\
\hline \hline
\end{tabular}

We also studied the performance of the receiver without neural network. We found that during certain combinations of training, the neural network based receiver yields better performance than the normal PIC receiver that requires more number of stages.

\section{CONCLUSION}

This work studied the effect of training parameters on the performance of neural network based receiver. We found that high volume of training data can bring better performance, without the requirement of high number of PIC stages. We also found that suitable selection of training parameters reduce the complexity of the PIC receiver. But in this work, we did not in-cooperate the multipath effect which plays a vital role in CDMA environment. Further modification can be based on the RAKE receiver based neural network receivers. This study thus points out the need of study on adaptive receivers based on neural network.

\section{ACKNOWLEDGMENT}

The authors wish to thank QualComm, Bangalore Division, India for their technical support in the fulfillment of this project.

\section{REFERENCES}

[1] B. Sklar, Digital Communications Fundamentals and Applications, 2nd ed. Prentice Hall, ch. 1-4, pp. 1-235, ch. 12, pp. 718-782.

[2] S. Verdu, "Minimum Probability of Error for Asynchronous Gaussian Multiple Access Channels," IEEE Trans. on Informn. Theory, vol. IT-32, no. 1, pp. 85-96, Jan. 1986.

[3] J. Proakis and M. Salehi, Digital Communications, 5th ed. Mc Graw-Hill Higher Education, 2001, ch. 12 - 16, pp. 762- 1078.

[4] B. Aazhang, B. P. Paris, and G. C. Orsak, "Neural Networks for Multi-user Detection in Code-Division Multiple-Access Communications," IEEE Trans. on Communications, vol. 40, no.7, pp. 1212 -1222, July 1992.

[5] U. Mitra and H. V. Poor, "Neural network Techniques for Multi-user Demodulation," in Proc. IEEE Int. Conf. Neural Networks, 1993, vol. 3, pp.1538-1543
[6] M. H. Yang, J. L. Chen, and P. Y. Cheng, "Successive Interference Cancellation Receiver with Neural Network Compensation in the CDMA Systems," in Conf. Record 34th Asilomar Conf. Signals, System and Computers, 2000, vol. 2, pp.1417 - 1420.

[7] D. Divsalar and M. K. Simon, "Improved Parallel Interference Cancellation for CDMA," in Proc. IEEE Trans. on Communications, vol. 46, no. 2, pp. 258-268, Feb. 1998.

[8] I. Tasminar. (December 2007). On Parallel interference cancellation with neural network in the CDMA systems. [Online]. Available: http://www.emo.org.tr/ekler/12f3b5c457a92ab_ek.pdf.

[9] J. D. V. Sarwate and M. B. Pursley, "Cross correlation properties of pseudorandom and related sequences," IEEE Engineering Profession General Topics for Engineers (Math, Science \& Engineering), May 1980, vol. 68, no.5, pp. 593-619.

[10] S. Haykin, Communication Systems, 4th ed. John Wiley \& Sons, Inc., 1994, ch. 7, pp. 479-508

[11] Z. Xie, R. T. Short, and C. I. Rushforth, "A Family of Suboptimum Detectors for Coherent Multiuser Communication," IEEE Journal on Selected Areas in Communications, vol. 8, no. 4, pp. 683 -690, May 1990.

[12] E. D. Sontag and H. J. Sussmann. On Back-propagation can give rise to spurious local minima even for networks without hidden layers. [Online]. Available: http://www.math.rutgers.edu/ sontag/FTP_DIR/complex_systems.pdf

[13] Y. Shrivastava and S. Dasgupta, "Convergence issues in perceptron based adaptive neural network models," in Proc. 25th Annu. Allerton Conf. Comm., Control and Computing, CSL and the Dept. of ECE, Univ. of Illinois, Sept, 1987, pp. 1133-1141.

[14] B. Widrow, J. M. McCool, M. G. Larimore, and C. R. J. Jr., "Stationary and nonstationary learning characteristics of the LMS adaptive filter," IEEE Engineering Profession; General Topics for Engineers (Math, Science \& Engineering), 1976, vol. 64, no. 8, pp. $1151-1162$.

[15] J. Shynk and S. Roy, "Convergence properties and stationary points of a Perceptrons Learning Algorithm," IEEE Engineering Profession; General Topics for Engineers (Math, Science \& Engineering), Oct. 1990, vol. 78, no. 10, pp. 1599-1604.

[16] H. D. Beale, Neural Network Design, PWS Pub., 1996, ch. 4, pp. 4.1 4.36

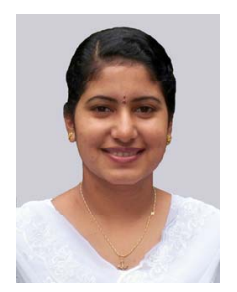

Jahan Rosemary Joseph has secured M. Tech. Degree in communication engineering and B.Tech. degree in electronics and communication engineering from MG University, Kottayam Kerala, during the years 2011 and 2009 respectively. Currently, she is working as Assistant Professor in the Department of Electronics and Communication Engineering, at Baselios Thomas I Catholicose College of Engineering and Technology, Kerala. She has attended conferences and presented papers in various international and national conferences. Her interested fields include Channel Modeling and Simulation, Wireless Communication and Interference Cancellation methods.

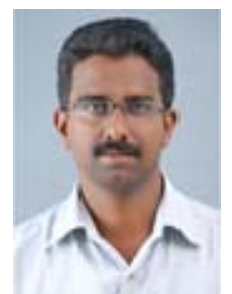

Anish Francis has secured M. Tech. degree in communication engineering from MG University, Kottayam, Kerala and B.Tech. Degree in electronics and communication engineering from Calicut University, Kerala in the years 2011 and 2001 respectively Previously, he was a Lecturer in the Department of Electronics and Communication Engineering, AJCE. Currently, he is working as Assistant Engineer, Kerala State Electricity Board, and Kerala. He has attended conferences and presented papers in various international and national conferences. His interested fields include Power Line Communication, Wireless Communication and Interference Cancellation using Neural Networks. 